\title{
Papers
}

\section{Co-proxamol and suicide: a study of national mortality statistics and local non-fatal self poisonings}

Keith Hawton, Sue Simkin, Jonathan Deeks

Centre for Suicide
Research, University
of Oxford
Department of
Psychiatry,
Warneford
Hospital,
Headington,
Oxford OX3 7JX
Keith Hawton
professor of psychiatry
Sue Simkin
researcher and
coordinator
Centre for Statistics
in Medicine,
Institute of Health
Sciences,
Headington,
Oxford OX3 7LF
Jonathan Deeks
senior medical
statistician
Correspondence to:
K Hawton
keith.hawton@
psych.ox.ac.uk

BMJ 2003;326:1006-8

\begin{abstract}
Objectives To examine the incidence of suicides due to co-proxamol compared with tricyclic antidepressants and paracetamol, and to compare fatality rates for self poisonings with these drugs. Design Analysis of routinely collected national and local data on suicides and self poisonings.

Setting Records of suicides in England and Wales 1997-9; non-fatal self poisonings in Oxford District 1997-9.

Data sources Office for National Statistics and Oxford monitoring system for attempted suicide. Main outcome measures Incidence of suicides with co-proxamol or tricyclic antidepressants or paracetamol. Ratios of fatal to non-fatal self poisonings.

Results Co-proxamol alone accounted for $5 \%$ of all suicides. Of 4162 drug related suicides, $18 \%$ (766) involved co-proxamol alone, 22\% (927) tricyclic antidepressants alone, and 9\% (368) paracetamol alone. A higher proportion of suicides in the 10-24 year age group were due to co-proxamol than in the other age groups. The odds of dying after overdose with co-proxamol was 2.3 times $(95 \%$ confidence interval 2.1 to 2.5 ) that for tricyclic antidepressants and 28.1 times (24.9 to 32.9) that for paracetamol. Conclusions Self poisoning with co-proxamol is particularly dangerous and contributes substantially to drug related suicides. Restricting availability of co-proxamol could have an important role in suicide prevention.
\end{abstract}

\section{Introduction}

Restriction of availability of means for suicide is a key strategy for prevention of suicide. ${ }^{12}$ This approach has been shown to be effective by the reduction in deaths after recent UK legislation that reduced pack sizes of analgesics ${ }^{3}$ and previous restriction of prescribing toxic sedatives. ${ }^{4}$

Co-proxamol is a prescription only analgesic that combines paracetamol and dextropropoxyphene. Respiratory depression and consequent death may occur with overdose due to ingestion of excessive dextropropoxyphene. ${ }^{56}$ Concern about the number of such deaths was expressed in the BMJ as long ago as $1980 .{ }^{7}$

We compared the numbers of suicides from poisoning with co-proxamol, paracetamol, and tricyclic antidepressants (these being relatively common methods in poisoning suicides) in England and Wales. We also compared fatal and non-fatal self poisonings to estimate the relative fatality of overdoses with these three drugs.

\section{Methods}

Mortality data-We obtained data from the Office for National Statistics on deaths in people aged 10 years and over in England and Wales for 1997-9. We considered those deaths that involved drugs and medicines in which a verdict of suicide (international classification of diseases, ninth revision, codes E950.0-E959.5) or undetermined cause (codes E980.0-E989.5) was recorded. Deaths involving co-proxamol, paracetamol, or tricyclic antidepressants were identified by searching for all variants of description of these drugs in the textual fields for cause of death.

Non-fatal self poisonings-Non-fatal self poisonings with co-proxamol, paracetamol or tricyclic antidepressants alone for 1997-9 were identified through the Oxford monitoring system for attempted suicide. ${ }^{8}$ This records all presentations to the general hospital in Oxford of deliberate self harm. The comprehensiveness and reliability of the data has previously been shown..$^{9}$ The pattern of drugs used for self poisoning in the Oxford area is similar to that seen elsewhere. ${ }^{10}$

Statistical analyses-We used Poisson regression to compute estimates, confidence intervals, and comparison of death rates and presentation rates according to drug, age, and sex. Odds ratios for the relative lethality of different drugs were calculated by computing ratios of relative death rates to relative non-fatal presentation rates. Confidence intervals for relative lethality were calculated with Monte Carlo methods. We used Stata release 7.0 (StataCorp, College Station, TX, USA) for the analyses.

\section{Results}

In England and Wales in 1997-9, 15299 deaths were recorded as suicides or open verdicts. Of these, 4162 (27\%) were drug related (such as self poisoning). There were more drug related deaths in men than in women, although a higher proportion of women who killed themselves did so by self poisoning (1835/3762 (49\%) $v 2327 / 11537$ (20\%) in men). 
Deaths due to self poisoning with co-proxamol There were 766 deaths due to poisoning with co-proxamol alone during the three year period (255 per year, $95 \%$ confidence interval 238 to 274). These comprised $18 \%$ of all drug related deaths (table 1) and $5 \%$ of all suicides. After tricyclic antidepressants co-proxamol was the second most common prescribed drug used for suicide. More co-proxamol poisoning deaths occurred in men than in women $(\mathrm{P}=0.01)$. Although the total numbers of deaths increased with age, the proportion due to co-proxamol poisoning was significantly higher $(24 \%)$ in $10-24$ year-olds $(\mathrm{P}=0.01)$. In addition, there were 171 deaths in which co-proxamol was used with another drug.

\section{Comparison with deaths due to tricyclic} antidepressants and paracetamol

There were an average 309 (289 to 330) deaths per year due to tricyclic antidepressants alone. These deaths comprised $22 \%$ of all drug related suicides, significantly more than for co-proxamol alone $(\mathrm{P}<0.001)$. Compared with men, women were more likely to use tricyclics than co-proxamol $(\mathrm{P}=0.02)$. Use of tricyclics was similar across age groups $(\mathrm{P}=0.07)$. In 270 deaths tricyclics were taken with other drugs.

An average 123 (110 to 136) deaths per year were due to paracetamol alone, and this comprised $9 \%$ of all drug related deaths (table 1). Compared with men, women were more likely to use paracetamol that co-proxamol $(\mathrm{P}=0.02)$, and its use increased more clearly with age $(\mathrm{P}<0.001)$. In 128 deaths paracetamol was taken with other drugs.

\section{Fatal and non-fatal self poisonings}

Table 2 gives details of annual numbers of non-fatal self poisonings. Non-fatal tricyclic antidepressant and paracetamol overdoses presented 2.7 (2.1 to 3.6) times and 13.5 (10.7 to 17.0$)$ times more frequently than non-fatal co-proxamol overdoses, respectively.

Comparison of ratios of death and non-fatal presentations suggests that the odds that an overdose will be fatal with co-proxamol is 2.3 (2.1 to 2.5) times higher than for tricyclic overdoses and 28.1 (24.9 to 32.9) times higher than for paracetamol overdoses.

\section{Discussion}

In England and Wales co-proxamol is the second most common prescribed drug that people use to commit suicide and is used as the sole method in $18 \%$ of all drug related suicides for $1997-9$ and $5 \%$ of all total suicides. Comparison with non-fatal poisonings indicates that overdoses of co-proxamol are more likely to result in death than overdoses with tricyclic antidepressant or paracetamol. Death can result from an overdose with relatively few tablets, especially when alcohol is also taken. ${ }^{5}$ Given earlier concerns about deaths from poi-
Table 1 Details of suicides by co-proxamol, tricyclic antidepressants, and paracetamol, England and Wales, 1997-9. Figures are numbers of suicides (percentage of all drug related suicides in age group), unless stated otherwise

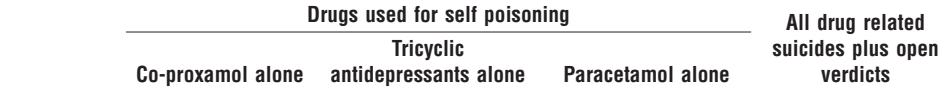

\begin{tabular}{|c|c|c|c|c|}
\hline & Co-proxamol alone & antidepressants alone & Paracetamol alone & verdicts \\
\hline \multicolumn{5}{|l|}{ Male } \\
\hline \multicolumn{5}{|c|}{ Age (years): } \\
\hline 10-24 & $55(23.8)$ & $53(22.9)$ & $8(3.5)$ & 231 \\
\hline 25-34 & $112(19.2)$ & $115(19.8)$ & $29(5.0)$ & 582 \\
\hline $35-54$ & 155 (15.8) & $221(22.6)$ & $77(7.9)$ & 978 \\
\hline$\geq 55$ & $97(18.1)$ & $63(11.8)$ & $59(11.0)$ & 536 \\
\hline Total & $419(18.0)$ & $452(19.4)$ & $173(7.4)$ & 2327 \\
\hline \multicolumn{5}{|l|}{ Female } \\
\hline \multicolumn{5}{|c|}{ Age (years): } \\
\hline 10-24 & $42(25.3)$ & $51(30.7)$ & $13(7.8)$ & 166 \\
\hline $25-34$ & 45 (14.6) & $72(23.4)$ & $29(9.4)$ & 308 \\
\hline $35-54$ & $118(17.2)$ & $176(25.6)$ & 75 (10.9) & 688 \\
\hline$\geq 55$ & $142(21.1)$ & $176(26.2)$ & 78 (11.6) & 673 \\
\hline Total & $347(18.9)$ & $475(25.9)$ & $195(10.6)$ & 1835 \\
\hline \multicolumn{5}{|l|}{ Total } \\
\hline \multicolumn{5}{|c|}{ Age (years): } \\
\hline $\begin{array}{c}10-24 \\
\end{array}$ & $97(24.4)$ & $104(26.2)$ & $21(5.3)$ & 397 \\
\hline $25-34$ & $157(17.6)$ & $187(21.0)$ & $58(6.5)$ & 890 \\
\hline $35-54$ & $273(16.4)$ & $397(23.8)$ & $152(9.1)$ & 1666 \\
\hline$\geq 55$ & $239(19.8)$ & $239(19.8)$ & $137(11.3)$ & 1209 \\
\hline Total & 766 (18.4) & $927(22.3)$ & $368(8.8)$ & 4162 \\
\hline
\end{tabular}

soning with to co-proxamol ${ }^{57}$ the absence of specific initiatives to try to reduce them is surprising and should now be addressed. ${ }^{1}$

Factors to be considered in tackling this problem are that co-proxamol is a prescription only drug, patients with pain already have an increased risk of suicide, ${ }^{11}{ }^{12}$ the risk of self poisoning is not restricted to the person for whom the drug is prescribed, ${ }^{13}$ and, as with paracetamol, ${ }^{14}$ availability within a household may be an influential factor, especially in impulsive overdoses. Also, while co-proxamol is regarded as an important analgesic, ${ }^{15}{ }^{16}$ a systematic review has shown that it is no more effective than paracetamol for short term relief of pain. ${ }^{17}$

Reducing the availability of drugs used for suicide can result in a reductions in deaths, ${ }^{3{ }^{4}}$ and availability of co-proxamol should be restricted. Clinicians must be informed about the risks of overdose of co-proxamol, both for their patients and others in the household, and large quantities should not be prescribed without good reason. Patients should be instructed to dispose of unwanted supplies. Finally, clinicians should consider whether there are other equally effective but less dangerous methods of pain relief, such as combining a safer analgesic with an opiate while maximal relief is required.

We thank Alan Baker of the Office for National Statistics, and Liz Bale, Alison Bond, and the staff of the Department of Psychological Medicine, John Radcliffe Hospital.

Table 2 Comparison of numbers (95\% confidence intervals) of drug related suicides and undetermined deaths in England and Wales with non-fatal self poisoning in Oxford, 1997-9, for co-proxamol, paracetamol, and tricyclic antidepressants (used alone). Odds ratios shown with $95 \%$ confidence intervals

\begin{tabular}{lccc} 
& Co-proxamol & Tricyclic antidepressants & Paracetamol \\
\hline Deaths in England and Wales/year & $255(238$ to 274$)$ & 309 (289 to 330) & 123 (110 to 136) \\
\hline Non-fatal self poisonings in Oxford/year & 26 (21 to 33) & 73 (64 to 83) & 356 (335 to 378$)$ \\
\hline Odds ratio for relative lethality compared with paracetamol & $28.1(24.9$ to 32.9$)$ & 12.3 (11.5 to 13.2) & 1.0 \\
\hline Odds ratio for relative lethality compared with tricyclics & $2.3(2.1$ to 2.5$)$ & 1.0 & 0.08 (0.08 to 0.09$)$ \\
\hline
\end{tabular}




\section{What is already known on this topic}

Co-proxamol is dangerous in overdose

Restricting availability of specific means of suicide can reduce deaths

\section{What this study adds}

Fatal overdoses due to co-proxamol are the second most frequent means of suicide with prescribed drugs in England and Wales

The risk of death associated with co-proxamol overdose seems to be higher than for either tricyclic antidepressants or paracetamol

Contributors: KH initiated the study, SS coordinated the data collection, and JD conducted the statistical analyses. All authors contributed to the study design, interpretation of the results, and preparation of the report. KH is guarantor for the study.

Funding: South East Region NHSE Research and Development Committee. $\mathrm{KH}$ is also supported by Oxfordshire Mental Healthcare NHS Trust. The guarantor accepts full responsibility for the conduct of the study, had access to the data, and controlled the decision to publish.

Competing interests: None declared.

1 Department of Health. National suicide prevention strategy for England. London: Department of Health, 2002.
2 Taylor SJ, Kingdon D, Jenkins R. How are the nations trying to prevent suicide? An analysis of national suicide prevention strategies. Acta Psychisuicide? An analysis of nation

3 Hawton K, Townsend E, Deeks JJ, Appleby L, Gunnell D, Bennewith O, et al. Effects of legislation restricting pack sizes of paracetamol and salicylates on self poisoning in the United Kingdom: before and after study. BMJ 2001;322:1203-7.

4 Oliver RG, Hetzel BS. Rise and fall of suicide rates in Australia: relation to sedative availability. Med J Aust 1972;2:919-23.

5 Committee on Safety of Medicines. Current problems 14. London: Committee on Safety of Medicines, 1985.

Dollery C. Therapeutic drugs. Edinburgh: Churchill Livingstone, 1999

Young RJ, Lawson AA. Distalgesic poisoning-cause for concern. BMJ Young RJ, Lawson

8 Hawton K, Fagg J, Simkin S, Bale E, Bond A. Trends in deliberate self-harm in Oxford, 1985-1995. Implications for clinical services and the prevention of suicide. BrJ Psychiatry 1997;171:556-60.

9 Sellar C, Goldacre MJ, Hawton K. Reliability of routine hospital data on poisoning as measures of deliberate self poisoning in adolescents. J Epidemiol Community Health 1990;44:313-5.

10 Bialas MC, Reid PG, Beck P, Lazarus JH, Smith PM, Scorer RC, et al. Changing patterns of self-poisoning in a UK health district. $O J M$ 1996;89:893-901.

11 Penttinen J. Back pain and risk of suicide among Finnish farmers. Am J Public Health 1995;85:1452-3.

12 Fishbain DA. The association of chronic pain and suicide. Semin Clin Neuropsychiatry 1999;4:221-7.

13 Hawton K, Houston K, Shepperd R. Suicide in young people: a study of 174 cases, aged under 25 years, based on coroners' and medical records. BrJ Psychiatry 1999;175:1-6.

14 Hawton K, Ware C, Mistry H, Hewitt J, Kingsbury S, Roberts D, et al. Why patients choose paracetamol for self poisoning and their knowledge of its dangers. $B M J$ 1995;310:164-4.

15 Haigh S. 12 years on: co-proxamol revisited. Lancet 1996;347:1840-1.

6 Sykes JV, Hanks GW, Forbes K. Co-proxamol revisited. Lancet 1996;348:408.

$17 \mathrm{Li}$ Wan Po A, Zhang WY. Systematic overview of co-proxamol to assess analgesic effects of addition of dextropropoxyphene to paracetamol. $B M J$ 1997;315:1565-71.

(Accepted 5 March 2003)

\section{ELPS}

This is an abridged version; the full version is on bmj.com

Office of Public

Policy and Ethics,

Institute for

Molecular

Bioscience,

University of

Queensland,

Brisbane,

Queensland 4072 ,

Australia

Wayne D Hall

professor and director

School of Public

Health and

Community

Medicine,

University of New

South Wales,

Sydney, New South

Wales 2052,

Australia

Andrea Mant

associate professor

Valerie A Rendle

research assistant

continued over

BMJ 2003;326:1008-11

\section{Association between antidepressant prescribing and suicide in Australia, 1991-2000: trend analysis}

Wayne D Hall, Andrea Mant, Philip B Mitchell, Valerie A Rendle, Ian B Hickie, Peter McManus

\begin{abstract}
Objective To examine the association between trends in antidepressant prescribing and suicide rates in Australia for 1991-2000.

Design Analysis of databases of suicide and rates of antidepressant prescribing according to age and sex. Setting Australian Bureau of Statistics data, sales data from the Australian pharmaceutical industry, prescribing data in general practice.

Subjects Men and women aged 15 years and over in 10 year age groups.

Main outcome measures Trends in suicide rates and trends in antidepressant prescribing. Association measured by Spearman's rank correlations.

Results While overall national rates of suicide did not fall significantly, incidence decreased in older men and women and increased in younger adults. In both men $\left(r_{s}=-0.91 ; \mathrm{P}<0.01\right)$ and women $\left(r_{s}=-0.76\right.$; $\mathrm{P}<0.05)$ the higher the exposure to antidepressants the larger the decline in rate of suicide.

Conclusions Changes in suicide rates and exposure to antidepressants in Australia for 1991-2000 are significantly associated. This effect is most apparent in older age groups, in which rates of suicide decreased substantially in association with exposure to
\end{abstract}

antidepressants. The increase in antidepressant prescribing may be a proxy marker for improved overall management of depression. If so, increased prescribing of selective serotonin reuptake inhibitors in general practice may have produced a quantifiable benefit in population mental health.

\section{Introduction}

In many developed countries the number of prescriptions for antidepressants increased steeply during the 1990s, after the introduction of selective serotonin reuptake inhibitors (SSRIs). ${ }^{1-4}$ In some countries, such as Sweden and Hungary, the increased rate of prescribing coincided with fall in the suicide rate..$^{2-4}$

We examined the association between antidepressant prescribing in Australia and changes in rates of suicide for 1991-2000. We analysed differences in suicide trends between men and women in different age groups to assess whether age and sex rates in suicide were related to exposure to antidepressant medication, or to a change in that exposure over time.

\section{Methods}

We used a quasi-experimental approach to analyse associations using prospectively collected data sets. ${ }^{5} \mathrm{We}$ 